\title{
On Surface Waves in Materials with Negative Poisson Ratio
}

\author{
P. Zieliński ${ }^{a, b}$, D. TharóG ${ }^{a, *}$ And D. TrzupeK ${ }^{c}$ \\ ${ }^{a}$ The H. Niewodniczański Institute of Nuclear Physics PAS \\ Radzikowskiego 152, 31-342 Kraków, Poland \\ ${ }^{b}$ Institute of Physics, Cracow Technical University \\ Podchorążych 1, 30-084 Kraków, Poland \\ ${ }^{c}$ Institute of Physics, Jagiellonian University \\ Reymonta 4, 30-059 Kraków, Poland
}

\begin{abstract}
True surface wave, i.e. a resonance with infinite lifetime is found within a bulk band in a model of thin planar layer coating a continuous auxetic substrate. Conditions for the existence of this wave are given.

PACS numbers:
\end{abstract}

\section{Introduction}

Auxetics are the materials that get fatter when stretched. This counterintuitive property is quantitatively rendered by a negative Poisson ratio. The latter is defined as the quotient of the linear perpendicular contraction to the expansion parallel to the axis of an applied uniaxial tensile stress. In fact, there can be two Poisson's ratios in low symmetry materials so that the material may expand in one direction perpendicular to the stress axis and at the same time shrink in another perpendicular direction. Materials are called full auxetics when both Poisson ratios are negative and auxetics if only one of them is negative. Negative Poisson ratio is found in crystals [1], in biological tissues [2] and in synthetic materials such as foams [3]. Particular interest for auxetics comes from medical engineering where such materials may serve as arterial prostheses [4], anchors for suture, muscles and ligaments, hip arthroplastic materials and media controlling release of healing agents [5].

\section{The model}

In this note we study the dynamics of a planar surface in an isotropic auxetic treated in the continuum approximation. All the elastic constants $C_{n m k l}$ of isotropic materials are expressed by two parameters: the Young modulus $E$ and the Poisson ratio $\sigma$ :

$$
\begin{aligned}
& C_{n m k l}=\frac{E}{(1+\sigma)} \\
& \quad \times\left[\frac{\sigma}{(1-2 \sigma)} \delta_{n m} \delta_{k l}+\frac{1}{2}\left(\delta_{n k} \delta_{m l}+\delta_{n l} \delta_{m k}\right)\right] .
\end{aligned}
$$

When free of external stresses, any isotropic material is mechanically stable if

$$
E>0 \text {, }
$$

and

$$
-1<\sigma<1 / 2
$$

All the acoustic waves in the isotropic media are either purely longitudinal or transverse. They propagate at the phase speeds $c_{\mathrm{L}}$ and $c_{\mathrm{T}}$, respectively

$$
\begin{aligned}
& c_{\mathrm{L}}=\sqrt{C_{1111} / \rho}=\sqrt{\frac{E}{\rho} \frac{(1-\sigma)}{(1+\sigma)(1-2 \sigma)}}, \\
& c_{\mathrm{T}}=\sqrt{C_{1212} / \rho}=\sqrt{\frac{E}{\rho} \frac{1}{2(1+\sigma)}} .
\end{aligned}
$$

Both phase speeds tend to infinity in the limit of stability $\sigma \rightarrow-1$, where the ratio $c_{\mathrm{T}} / c_{\mathrm{L}}$ takes its maximum value $1 / \sqrt{2}$. In systems subject to an external stress the Poisson ratio has no limitation [6]. The increase in the elastic stretch $C_{1111}$ and shear $C_{1212}$ moduli with increasing negative Poisson ratio have important technological consequences.

In the half space $x_{3}>0$ occupied by the material the vibrations of the medium with a planar surface satisfy the Christoffel equations of motion

$$
\rho \frac{\partial^{2} u_{n}}{\partial t^{2}}=\sum_{m, k, l=1}^{3} C_{n m k l} \frac{\partial^{2} u_{k}}{\partial x_{m} \partial x_{l}},
$$

where $u_{n}, n=1, \ldots 3$, are components of displacement. The surface itself imposes appropriate boundary conditions. In the present work the conditions read

$$
\rho_{\mathrm{s}} \frac{\partial^{2} u_{1}}{\partial t^{2}}=E_{\mathrm{s}} \frac{\partial^{2} u_{1}}{\partial x_{1}^{2}}+\frac{E}{1+\sigma}\left(\frac{\partial u_{1}}{\partial x_{3}}+\frac{\partial u_{3}}{\partial x_{1}}\right),
$$

\footnotetext{
* corresponding author; e-mail: Dariusz.Twarog@ifj.edu.pl
} 


$$
\begin{aligned}
& \rho_{\mathrm{s}} \frac{\partial^{2} u_{3}}{\partial t^{2}}=A_{\mathrm{s}} \frac{\partial^{2} u_{3}}{\partial x_{1}^{2}}+\frac{E}{(1+\sigma)(1-2 \sigma)} \\
& \quad \times\left[(1-\sigma) \frac{\partial u_{3}}{\partial x_{3}}+\sigma \frac{\partial u_{1}}{\partial x_{1}}\right] .
\end{aligned}
$$

They model an infinitely thin layer of planar density $\rho_{\mathrm{S}}$ of the stretch modulus in the surface plane $E_{\mathrm{s}}$ and of the surface bending modulus $A_{\mathrm{s}}$. The latter quantity is usually neglected in the theory of thin plates [7] but it must be taken into account for some metamaterials. In fact, many auxetic materials are artificial and are composed of some rigid elements [1-6]. The same may concern the surface layer.

\section{Results}

Figure 1 presents an example of a material in which the bending modulus clearly exceeds the stretch modulus. Such examples motivate the use of the first term on the right hand side of Eq. (8). The reference system is chosen so that the $x_{1}$ axis lies along the wave vector component parallel to the surface, whereas $x_{3}$ the direction perpendicular to the surface into the bulk material. For the moment only saggital waves are considered.

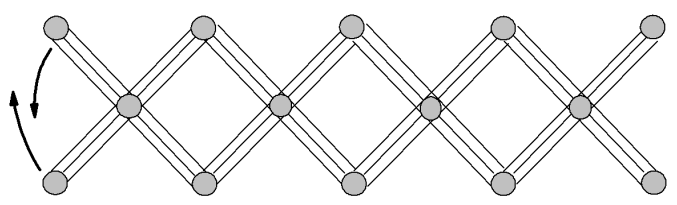

Fig. 1. Structure (Nuernberg scissors) of rigid rods connected by articulations indicated by grey circles. Structure is easy to stretch but hard to bend. Thus, bending modulus exceeds Young's modulus.

General solution of the equations of motion (Eq. (6)) with the boundary conditions (Eqs. (7) and (8)) have a form of waves

$$
\begin{array}{r}
u_{i}\left(x_{1}, x_{3}, t\right)=A^{\mathrm{L}} u_{i}^{\mathrm{L}} \exp \left(-\mathrm{i} \omega t+k_{1} x_{1}+k_{3}^{\mathrm{L}} x_{3}\right) \\
+A^{\mathrm{T}} u_{i}^{\mathrm{T}} \exp \left(-\mathrm{i} \omega t+k_{1} x_{1}+k_{3}^{\mathrm{T}} x_{3}\right), i=1,3,
\end{array}
$$

where the components of the unit vectors $u^{\mathrm{L}}$ and $u^{\mathrm{T}}$ satisfy the relations $u_{3}^{\mathrm{L}} / u_{1}^{\mathrm{L}}=-k_{1} / k_{3}^{\mathrm{T}}$ and $u_{3}^{\mathrm{L}} / u_{1}^{\mathrm{L}}=k_{3}^{\mathrm{L}} / k_{1}$, while the components of the wave vectors perpendicular to the surface $k_{3}^{\mathrm{L}}$ and $k_{3}^{\mathrm{T}}$ are defined by the dispersion relations $\omega^{2}=c_{\mathrm{L}}^{2}\left[k_{1}^{2}+\left(k_{3}^{\mathrm{L}}\right)^{2}\right]$ and $\omega^{2}=c_{\mathrm{T}}^{2}\left[k_{1}^{2}+\left(k_{3}^{\mathrm{T}}\right)^{2}\right]$. The regions where $k_{3}^{\mathrm{L}}$ or $k_{3}^{\mathrm{T}}$ are real are called bulk bands. The solutions of Eqs. (7) and (8) for the amplitudes $A^{\mathrm{L}}$ and $A^{\mathrm{T}}$, if they exist at all, then show complex frequencies $\omega=\operatorname{Re} \omega-\mathrm{i} \operatorname{Im} \omega$. Such solutions describe surface resonances and the quantities $1 / \operatorname{Im} \omega$ correspond to their lifetimes. Outside the bulk bands, where both $k_{3}^{\mathrm{L}}$ and $k_{3}^{\mathrm{T}}$ are purely imaginary, any possible solution of Eqs. (7) and (8) with real frequency corresponds to a true surface wave.

An interesting property of the present model is that at specific parameters of the surface and of the bulk a true surface wave, i.e. a resonance with infinite lifetime may occur within bulk band. The conditions for the existence of this exceptional surface wave (ESW) can be found by inserting the wave ansatz (Eq. (9)) into Eqs. (7) and (8) in the region where $k_{3}^{\mathrm{T}}$ is real and $k_{3}^{\mathrm{L}}$ is imaginary and by requiring that $A^{\mathrm{T}}=0$. Then the wave is a real surface wave, although its frequency is placed in the bulk band of transverse waves. The system of equations resulting from the above conditions reads

$$
\begin{aligned}
& \frac{1-\sigma^{2}}{2 \sigma}\left(\gamma_{\mathrm{ES}}-\gamma_{\mathrm{AS}}\right)\left(d \kappa_{\mathrm{L}}\right)^{2}+\frac{1+\sigma}{2 \sigma} \\
& \quad \times\left[\frac{1-\sigma}{1+\sigma}-2 \gamma_{A S}(1-2 \sigma)+\gamma_{E S}(1-\sigma)\right] d \kappa_{\mathrm{L}}+1 \\
& \quad=0
\end{aligned}
$$

and

$$
\left(k_{1} d\right)^{2}=\frac{1}{1-c_{\mathrm{ls}} / c_{\mathrm{L}}^{2}}\left[\left(\kappa_{\mathrm{L}} d\right)^{2}+2 \kappa_{\mathrm{L}} d\left(\frac{1-2 \sigma}{1-\sigma}\right)\right],
$$

where $\gamma_{\mathrm{ES}}=\frac{E_{\mathrm{s}} \rho}{E \rho_{\mathrm{s}}}, \gamma_{\mathrm{AS}}=A_{\mathrm{s}} \rho / E \rho_{\mathrm{s}}$ and $d=\rho_{\mathrm{s}} / \rho$ is the layer thickness.

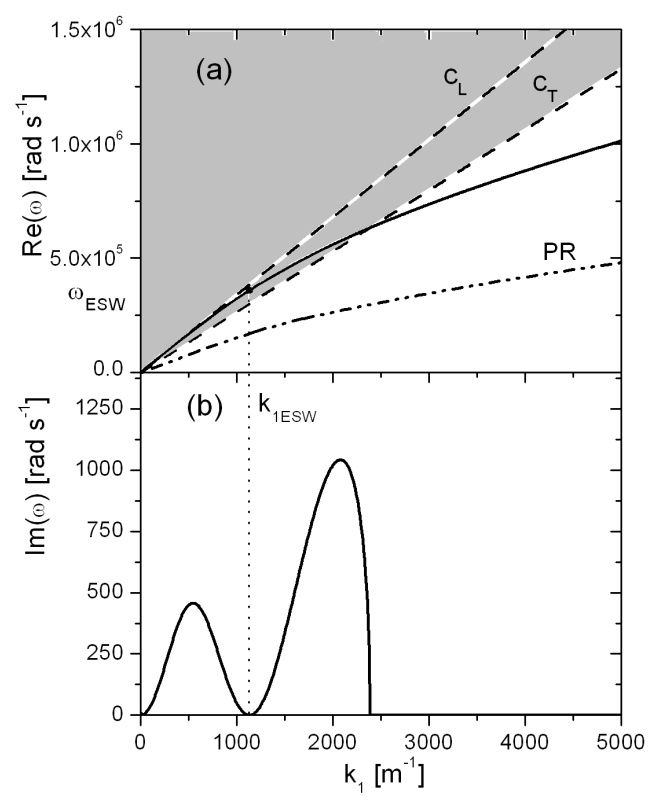

Fig. 2. Bulk band (gray) and dispersion curves of surface waves at presence of ESW (a). Imaginary part of frequency along dispersion curve (continuous line in upper panel) of surface waves and resonances where ESW occurs (b).

Figure 2 shows the bulk bands and the dispersion curves of the surface waves in the present model with parameters ensuring the ESW. The Poisson ratio of the bulk medium is negative. The imaginary part of the frequency of the surface resonance within the bulk band of transverse waves vanishes at the point of ESW. A consequence of a narrow resonance within the bulk band is that the reflection coefficient of the transverse wave coming from the bulk undergoes strong variation. This is seen in Fig. 3. A very strong reflection coefficient cor- 


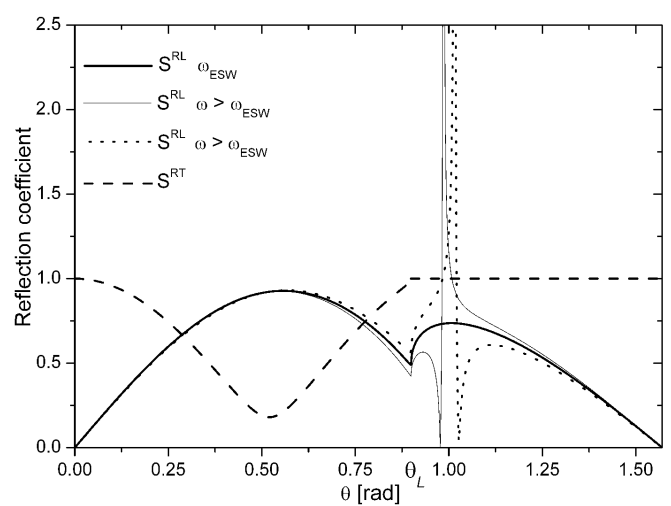

Fig. 3. Amplitude reflection coefficients of reflected transverse $\left(S^{\mathrm{RT}}\right)$ and longitudinal $\left(S^{\mathrm{RL}}\right)$ waves as functions of incidence angle of transverse incident wave polarized in incidence plane for three frequencies close to that corresponding to ESW. The reflection coefficient $S^{\mathrm{RT}}$ lies practically on the same curve in all the cases.

responds to the incoming wave being practically directed to the surface. Practical consequences of ESW are under studies.

\section{Acknowledgments}

This work is part of project "Ion and plasma nanotechnologies for materials formed on the basis of C and Si" in the frame of operational program Innovative Economy (Poland). The National Network MANAR of the Ministry MNiSW (Poland) has provided a significant support. The contribution of the cooperation project of Polish Academy of Sciences and Centre National de la Recherche Scientifique (France) is acknowledged.

\section{References}

[1] K.E. Evans, M.A. Nkansh, I.J. Hutchinson, S.C. Rogers, Nature 353, 124 (1991).

[2] J.I. Williams, R.A. Lewis, J. Biomech. Eng. 104, 50 (1982).

[3] R.S. Lakes, Nature 1038, 235 (1987).

[4] B.D. Caddok, K.E. Evans, Biomaterials 16, 1109 (1995).

[5] See Auxetics Technologies Ltd., www.auxetictechnologies.com.

[6] D, Trzupek. D. Twaróg, P. Zieliński, Acta Phys. Pol. A 115, 576 (2009).

[7] L.D. Landau, E.M. Lifschitz, Lehrbuch Der Theoretischen Physik, translated by P. Ziesche, AkademieVerlag, Berlin 1981. 